\section{Acute Presentation of Tophaceous Myelopathy}

\section{To the Editor:}

Tophaceous gout of the axial skeleton is an unusual presentation. Most cases occur in men with a history of severe polyarticular gout ${ }^{1}$. In reported cases, patients have usually presented with symptoms including neck and back pain, radiculopathy, myelopathy, and cauda equina syndrome ${ }^{2,3}$. To our knowledge, this is the first reported case of spinal gout presenting with acute paraplegia.

A 34-year-old man with a history of morbid obesity, diabetes mellitus, and renal insufficiency presented with acute onset of motor and sensory loss below the level of his nipples. He awoke early that morning noting leg weakness. While walking to the bathroom, he fell, after which he lost sensation in his legs and was unable to urinate. He had no history of fevers or prior neuromuscular symptoms. His medications on admission included loperimide, aripiprazole, zolpidem, trazodone, and simvastatin. He had been treated intermittently for gout with allopurinol, most recently reinstituted 9 days prior to his fall.

On examination, the patient was morbidly obese, with body mass index of 69.5. Vital signs showed blood pressure 124/68 mm Hg, heart rate $112 \mathrm{bpm}$, and temperature $37.7^{\circ} \mathrm{C}$. He was alert and oriented and his pupils were equally reactive to light. Motor strength was $5 / 5$ in the upper extremities and $0 / 5$ in the lower extremities. Sensation to pinprick was absent below the T6 dermatome. Proprioception and reflexes were absent in bilateral lower extremities. Joint examination revealed no swollen joints or tophi.

Laboratory results showed white blood cell count $7300 / \mathrm{mm}^{3}$, hemoglobin $11.3 \mathrm{~g} / \mathrm{dl}$, blood urea nitrogen $16 \mathrm{mg} / \mathrm{dl}$, and creatinine $1.8 \mathrm{mg} / \mathrm{dl}$. Erythrocyte sedimentation rate was $54 \mathrm{~mm} / \mathrm{h}$, C-reactive protein $195 \mathrm{mg} / \mathrm{l}$, and serum uric acid $7.3 \mathrm{mg} / \mathrm{dl}$. Computed tomography scan of the spine showed multilevel osteophytes as well as spinal stenosis from T8 to L1. Magnetic resonance imaging (MRI) showed cord compression from an epidural fluid collection from T1 to T5 (Figure 1). He was started on empiric antibiotics for presumed epidural abscess and he was sent to surgery for a thoracic laminectomy from T2 to T10. Epidural fluid collection showed moderate polymorphonuclear leukocytes on Gram stain, but no growth of microorganisms by aerobic or anaerobic culture. Pathological analysis of the laminectomy specimen (Figure 2) confirmed the presence of gout.

Over the next several days, the patient complained of swelling and pain in his right elbow and developed a fever of $39.1^{\circ} \mathrm{C}$. Aspiration of the right olecranon bursa produced $0.75 \mathrm{cc}$ of thick curdish fluid, which was Gram stain and culture-negative. Polarized microscopy of the aspirate showed negatively birefringent crystals and numerous polymorphonuclear leukocytes. He also developed pain and swelling in his left elbow as well as his left wrist. He received corticosteroid injections to bilateral olecranon bursae and the left wrist, and was started on prednisone $60 \mathrm{mg}$ daily. After 1 week of treatment, his prednisone dose was tapered $10 \mathrm{mg}$ per day. He was then started on colchicine prophylaxis (0.6 mg daily) and transitioned to allopurinol as an outpatient. Postoperatively, he recovered sensory but not muscular function in his lower extremities.

Common locations for gouty tophi include joints, pressure areas of the forearms, and bursae. Tophaceous deposits in the axial skeleton are rare. Our patient's initial leg weakness and flaccid paralysis was likely due to an inflamed gouty tophus compressing the thoracic spinal cord. His ensuing fall resulted in rupture of the tophus, leading to increased pressure on the cord and paraplegia.

Radiographic findings of spinal gout include vertebral erosions, bone destruction causing joint subluxation, pathologic fractures, and osteophyte formation ${ }^{4}$. MRI findings include abnormal signals in T1- and T2-weighted images representing the gouty tophus ${ }^{4}$. However, these findings are nonspecific and can also be seen in cases of central nervous system infection. Histologic features of tophaceous gout include granuloma formation with histiocytes, fibroblasts, and multinucleated giant cells surrounding hypocellular amorphous material.

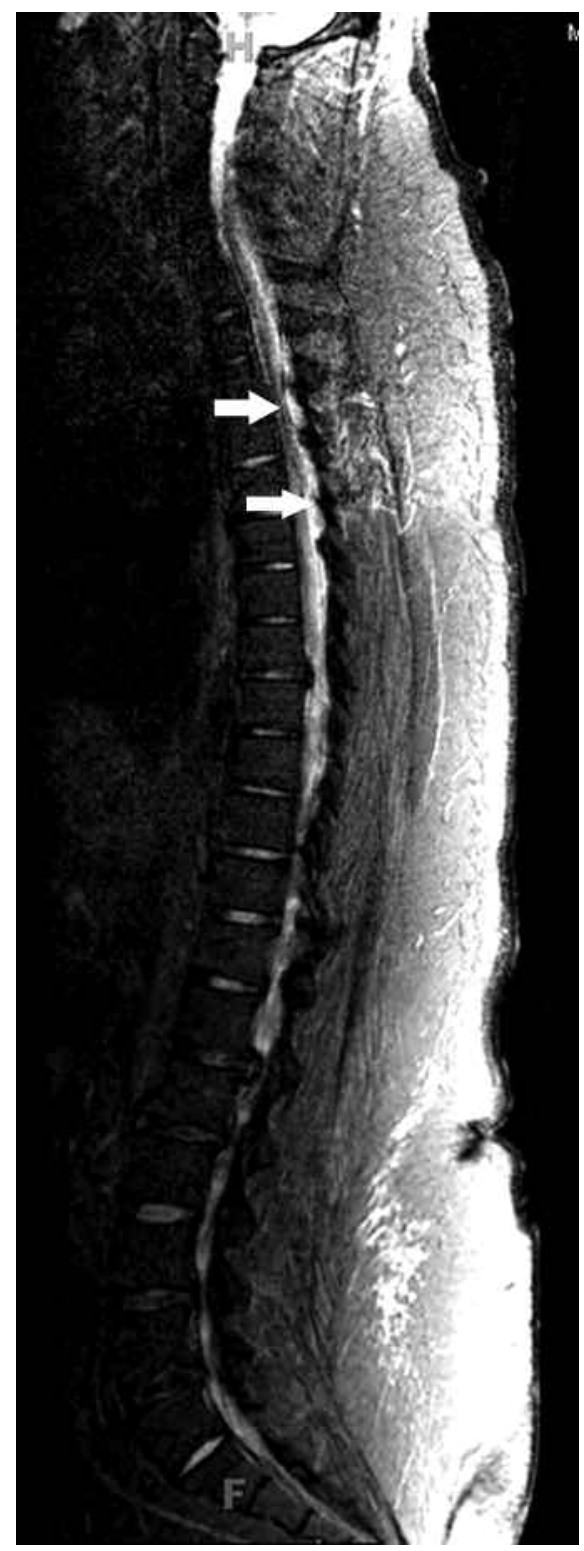

Figure 1. T2 short-tau inversion recovery MRI of the cervical spine without contrast. Arrows outline area of cord compression from epidural fluid collection.

Treatment of spinal gout leading to cord compression requires emergent laminectomy ${ }^{5}$. If the patient complains only of back pain in the setting of polyarticular tophaceous gout, medical management with nonsteroidal antiinflammatory drugs, intraarticular or systemic corticosteroids, or colchicine is appropriate to forestall the acute attack $^{5}$. Longterm management includes a uric acid-lowering medication such as allopurinol to dissolve tophaceous deposits and prevent further attacks.

This case emphasizes the importance of including gout in the differential diagnosis of a myelopathy of unknown etiology. Clinical suspicion should be increased in the setting of a history of gout. Diagnosis can lead to initiation of appropriate and timely therapy and improve patient outcomes by reducing pain from the gout flare. It also reduces the potential harm of unneeded antibiotics or exploratory surgery. 


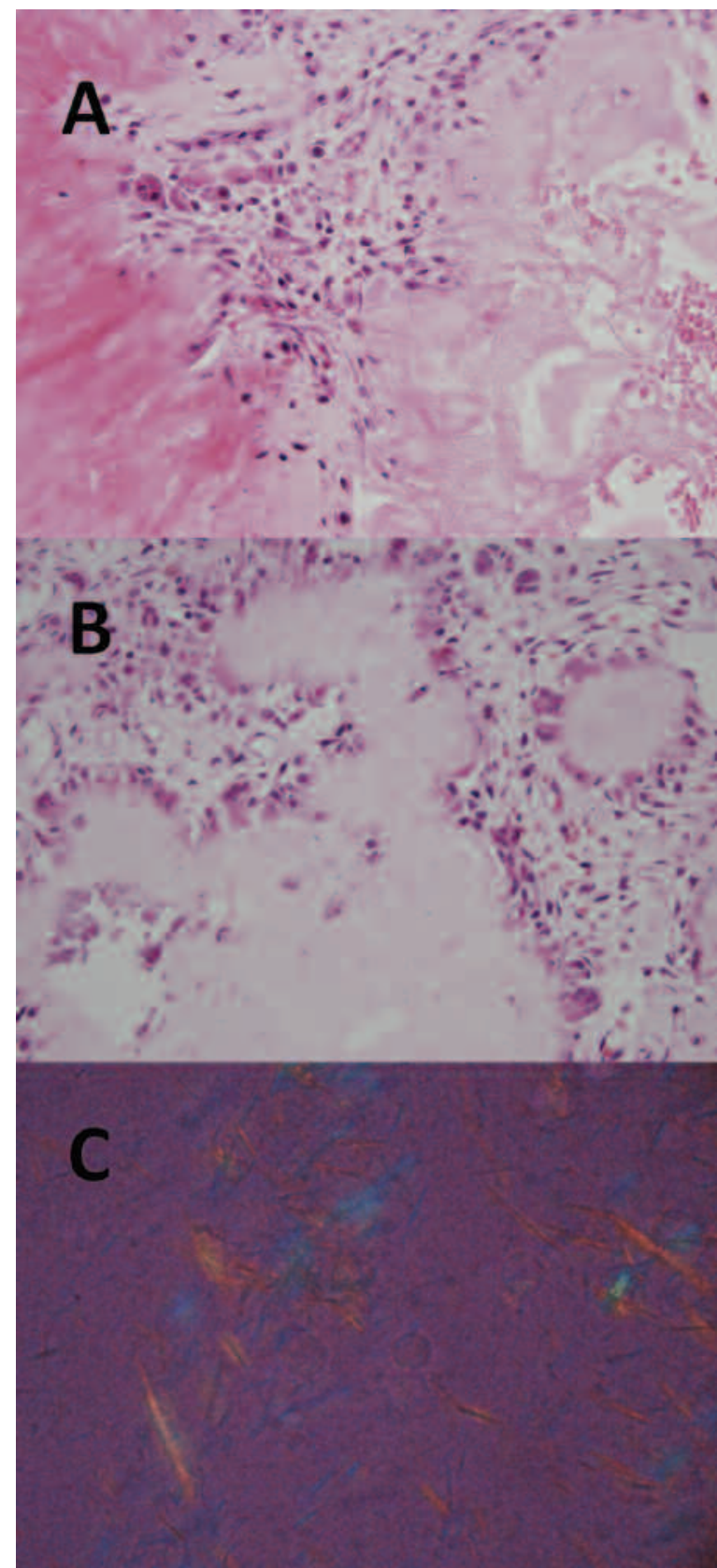

Figure 2. Laminectomy specimen (A and B) and olecranon bursa aspirate specimen (C). A. High power view (40× objective) showing amorphous pale eosinophilic material (right side) separated from necrotic cartilage (left side), by a mononuclear infiltrate containing prominent macrophages and fibroblasts. B. High power view ( $40 \times$ objective) of amorphous pale eosinophilic material surrounded by multinucleated giant cells and reactive-appearing periosteum. C. Polarized microscopy of aspirate specimen showing negatively birefringent crystals.
ETHAN LEVIN, MD, Department of Medicine; KYLE HURTH, MD, PhD, Department of Pathology and Immunology; REETI JOSHI, MD; RICHARD BRASINGTON, MD, FACP, Department of Medicine, Washington University School of Medicine, 660 S. Euclid Campus, Box 8045, St. Louis, Missouri 63110, USA.

The authors thank Dr. Matthew Parsons, Assistant Professor of Radiology, Mallinckrodt Institute of Radiology, Washington University School of Medicine, for providing radiological images.

\section{REFERENCES}

1. Ko KH, Huang GS, Chang WC. Tophaceous gout of the lumbar spine. J Clin Rheumatol 2010;16:200.

2. Barrett K, Miller ML, Wilson JT. Tophaceous gout of the spine mimicking epidural infection: Case report and review of the literature. Neurosurgery 2001;48:1170-2.

3. Jacobs SR, Edeiken J, Rubin B, DeHoratius RJ. Medically reversible quadriparesis in tophaceous gout. Arch Phys Med Rehabil 1985;66:188-90.

4. Bonaldi VM, Duong H, Starr MR, Sarazin L, Richardson J. Tophaceous gout of the lumbar spine mimicking an epidural abscess: MR features. Am J Neuroradiol 1996;17:1949-52.

5. Dhote R, Roux FX, Bachmeyer C, Tudoret L, Daumas-Duport C, Christoforov B. Extradural spinal tophaceous gout: evolution with medical treatment. Clin Exp Rheumatol 1997;15:421-3.

J Rheumatol 2011;38:7; doi:10.3899/jrheum.101335 